\title{
Variations
}

Variations

Revue internationale de théorie critique

$17 \mid 2012$

Critique du travail

\section{Pour un socialisme vert}

Extraits de Pour un socialisme vert, vers la société écologique par la justice sociale. Contribution à la critique de l'écologie politique

\section{Arno Münster}

\section{(2) OpenEdition}

\section{Journals}

Édition électronique

URL : http://journals.openedition.org/variations/419

DOI : 10.4000/variations.419

ISSN : 1968-3960

Éditeur

Les amis de Variations

Référence électronique

Arno Münster, «Pour un socialisme vert », Variations [En ligne], 17 | 2012, mis en ligne le 15 octobre 2012, consulté le 19 avril 2019. URL : http://journals.openedition.org/variations/419 ; DOI : 10.4000/ variations. 419

Ce document a été généré automatiquement le 19 avril 2019

Les ami•e•s de Variations 


\section{Pour un socialisme vert}

Extraits de Pour un socialisme vert, vers la société écologique par la justice sociale. Contribution à la critique de l'écologie politique

\section{Arno Münster}

\section{NOTE DE L'ÉDITEUR}

Variations remercie l'auteur et les Nouvelles Éditions Lignes de lui permettre de publier ces extraits de l'ouvrage Pour un socialisme vert, vers la société écologique par la justice sociale. Contribution à la critique de l'écologie politique, 2012, Fécamp, Nouvelles Éditions Lignes, 139 p., http://www.editions-lignes.com/MUNSTER-SOCIALISME-VERT.html

\section{Préambule sur « l'impossible capitalisme vert » (pp. 13-26)}

Dans son livre L'Impossible capitalisme vert, Daniel Tanuro ${ }^{1}$ met pour ainsi dire les points sur les « $\mathrm{i}$ » en résumant la situation paradoxale dans laquelle se trouvent nos sociétés contemporaines, à l'ère de la crise bancaire mondiale et de la globalisation néo-libérale : "Nous vivons dans une société que les élites prétendent gouverner selon "la raison", mais l'humanité, même dans les périodes les plus obscurantistes de son histoire, n'a sans doute jamais connu de situation aussi totalement, aussi monstrueusement "irrationnelle" "! Irrationnelle parce que, à peu près deux siècles après l'instauration par la Révolution Française du système économico-politique libéral issu de la chute de la Monarchie absolutiste, autrement dit du système fondé sur la raison, la liberté individuelle de l'entreprise, la propriété privée, le productivisme industriel et le profit, celui-ci semble avoir atteint ses limites. Cela, après avoir été régulièrement ébranlé dans ses fondements par des crises internes graves et toujours "sauvé» miraculeusement par des mesures et des interventions étatiques exceptionnelles, destinées à prolonger artificiellement son existence (1929-2008). Cet état de crise permanent n'a manifestement pas épargné le noyau même de sa "rationalité », et il semble plutôt confirmer la vision pessimiste 
annoncée à la fin des années 1940 par Adorno et Horkheimer. Au terme d'un long processus de réflexion philosophico-sociologique sur les Lumières et les chances historiques de réalisation de leur projet rationnel, les pères fondateurs de l'École de Francfort menèrent en effet la critique de la rationalisation excessive de nos sociétés modernes et entreprirent de s'intéresser au phénomène du « devenir-irrationnel » de la raison, à savoir de l'inversion de la raison en déraison, déjà immanente au processus initial de son déploiement.

Certes, les pères fondateurs de l'École de Francfort ${ }^{3}$ n'évoquaient alors guère l'écologie. Mais depuis la catastrophe nucléaire de Three Miles Island aux États-Unis (1979), la catastrophe industrielle de Bhopal (1984), en Inde, et évidemment celle de Tchernobyl (1986), l'écologie s'est imposée, dans le courant de la première décennie du siècle, et encore davantage après l'accident nucléaire de Fukushima, au Japon (mars 2011), comme l'une des toutes premières références théorico-politiques de nos débats et controverses politiques. Pourquoi? Parce que deux cents ans de productivisme ont mené le climat mondial au bord de «l'infarctus», et que la réalité nous impose désormais de réduire radicalement et très vite les émissions de gaz à effet de serre, et par voie de conséquence, notre production matérielle. Mais la mise en œuvre d'une politique de la décroissance, ne risque-t-elle pas de léser en même temps "le droit légitime au développement de celles et de ceux qui n'ont rien et qui sont en même temps les principales victimes du réchauffement ${ }^{4}$ ", comme le souligne à juste titre Daniel Tanuro?

Le capitalisme sera-t-il encore à même de résoudre cette crise, de nous tirer de ce dilemme? N'a-t-il pas déjà trop montré dans le passé qu'il ne sait nous proposer que des pseudo-remèdes, des pseudo-solutions, à savoir des pseudo-stabilisations d'un système économique fondé essentiellement sur le productivisme, la croissance et le profit, et qui est sûrement condamné, à long terme, à courir inexorablement vers l'abîme, précisément à cause de la fragilité extrême d'un de ses principaux piliers : le système bancaire. Sauvé de justesse de la débâcle totale en octobre 2008, ce dernier n'est pas vraiment en mesure, comme le montre la présente crise monétaire, de gérer à court ou à long terme les conséquences néfastes du surendettement des États et des ménages, ainsi que les déséquilibres générés par la faillite financière des États les plus fragilisés. Or, l'écroulement probable et possible, par effet de domino, des banques, à l'échelle mondiale, à cause de la faillite d'un ou de plusieurs États-Nations surendettés, incapables de rembourser leur dette, produirait à coup sûr un désastre tel que l'équilibre économicosocial de la plupart des pays de la zone euro, et non plus seulement celui de la Grèce, du Portugal, de l'Espagne et de l'Irlande, serait sérieusement menacé. Et on voit mal comment les gouvernements nationaux respectifs, contraints de gérer cette situation tout à fait impossible, comme le capitaine du «Titanic », pourraient encore trouver la solution miracle pour éviter le pire à leurs concitoyens en matière d'austérité, de chômage, de pauvreté, d'inflation, etc.

Avec la crise grecque du printemps 2011 (risque de faillite de l'État!), chacun a pu se rendre compte que l'austérité, avec toutes ses conséquences néfastes, imposée avec cynisme par le capitalisme financier (Le FMI, les agences de notation, la Banque Centrale Européenne et les dirigeants des principaux pays créanciers : l'Allemagne et la France) à un pays, accusé d'avoir vécu de manière " irresponsable », systématiquement " au-dessus de ses moyens", et exhorté sans cesse de se serrer la ceinture, ne sera pas limitée à ce seul pays, mais probablement étendue tôt ou tard à tous ceux de la zone "Euro", frappant ainsi également les populations des "pays riches» connaissant encore une 
stabilité économique relative, à savoir celles de l'Italie, de la France et de l'Allemagne. L'austérité généralisée, avec son cortège de chômage, de pauvreté, de déclin des classes moyennes, de montée des violences urbaines. En bref, ce scénario d'horreur pourrait un jour sonner le glas d'un système qui, courant de crise en crise, continue obstinément à refuser de changer de logique en dépit de tous les désastres qui se sont déjà produits, et qui, ne tirant aucune leçon sérieuse des crises précédentes, continue de privatiser les gains tout en socialisant les pertes. Les principales caractéristiques de cette crise sont que a) le crédit occupe aujourd'hui la place que le salaire tenait durant la période fordiste et b) «les profits demeurent, voire s'amplifient, mais le chômage et les inégalités ne cessent de croitre, les services publics sont détruits, les droits sociaux quotidiennement attaqué5. » Évidemment, sur le plan économique, la perspective est dans le meilleur des cas, comme le souligne François Chesnais, "celle d'une longue période de croissance mondiale à des taux moyens faibles, très faibles dans le cas de l'UE. [...] Les ressorts de l'accumulation des dix dernières années [...] sont cassés. Peu de pays échapperont à la quasi-stagnation ${ }^{6}$.» Et la perspective pour les pays hautement industrialisés de la zone Nord pourrait bien être une dépression profonde de longue durée. En revanche, les taux de croissance très élevés des pays émergents (Brésil, Inde, Chine) provoqueront une augmentation de plus en plus dangereuse de la pollution de l'air mettant en péril la vie de dizaines de millions d'êtres humains. Face à cela, l'offensive des climato-sceptiques (Claude Allègre...) a été déclenchée en France pour décrédibiliser les écologistes qui, à partir de données scientifiques incontestables (les recommandations du $\mathrm{GIEC}^{7}$ ), ont alerté l'opinion publique sur les effets désastreux des émissions à effet de serre, de la catastrophe climatique, de la fonte de la calotte polaire, des inondations et de l'asphyxie des grandes villes par des taux d'émission de $\mathrm{CO}_{2}$ devenus absolument insupportables.

À ce propos, l'un des mérites de Daniel Tanuro est d'avoir montré que la dégradation climatique ne peut être dissociée du fondement naturel du capitalisme, et qu'il est douteux de penser que des mesures prises par le capitalisme vert, telles que l'introduction d'une éco-taxe frappant les grands pollueurs, puissent effectivement renverser la tendance. Michel Husson considère quant à lui que «l'ampleur des mutations nécessaires est le fondement objectif de la convergence écosocialiste ${ }^{8} »$.

Autrement dit: dans une situation où la croissance des forces productives est devenue croissance des forces destructrices, la perspective des catastrophes à prévoir si l'on continue sur cette voie nous impose d'envisager et d'entamer une alternative au système dans son ensemble. Comme ce dernier a apparemment atteint sa limite et se trouve dans une impasse, pourquoi ne pas envisager sa sortie pure et simple? Or, comme nous l'a montré André Gorz, la seule alternative réelle à la catastrophe dans laquelle le capitalisme avancé nous a conduits, est et demeure l'alternative écosocialiste. Car elle seule sera à même, comme l'affirme encore Daniel Tanuro, de " tracer les lignes de force d'une issue rationnelle de la crise combinant quatre mouvements simultanés :1) la satisfaction des besoins sociaux réels, 2) la réduction de la production globale par la diminution du temps de travail, 3) la substitution des énergies fossiles par les énergies renouvelables et 4) l'institution politique d'une planification collective d'une économie convertie écologiquement ${ }^{9}$. » Ces quatre mouvements ne sont que le résultat d'une synthèse opérée entre les principales revendications d'une "écologie de combat » et les principaux objectifs du socialisme (égalité, liberté, justice sociale), donc d'une sorte de "mariage de raison" entre deux courants et tendances politiques revendiquant prioritairement une transformation économique, sociale et écologique radicale, au nom précisément du sauvetage de l'environnement et de la nature et au nom de 
la justice sociale. Il s'agit aussi de prendre enfin les mesures nécessaires pour limiter et juguler les pouvoirs abusifs de la finance, de la spéculation et de toute une oligarchie financière et industrielle ne connaissant que la loi de la valeur, du profit et de l'accumulation ${ }^{10}$. De toute façon, «la crise écologique, dont le changement climatique est l'élément central des conditions "non librement choisies" que le capitalisme laissera en héritage aux générations qui viennent, ne peut qu'aggraver la crise du capitalisme et précipiter son déclin. Par conséquent, "faire l'histoire", c'est essayer de devancer les catastrophes et en tous les cas de mettre la question écologique au cœur du projet révolutionnaire renouvelé1. ${ }^{11}$. Il s'agit en réalité d'aller beaucoup plus loin que Hans Jonas qui, dans son Principe Responsabilité ${ }^{12}$, interpelle les responsables politiques pour qu'ils tirent les conséquences de l'urgence écologique dans laquelle se trouve la planète, en nous proposant une sorte de "gouvernement mondial " des élites. Il s'agit plutôt de combiner, d'articuler les luttes écologiques et les luttes sociales, et, comme l'écrit François Chesnais, d'« inscrire la question écologique au cœur du programme révolutionnaire $\mathrm{du} \mathrm{XXI}^{e}$ siècle ${ }^{13}$ ", précisément dans la continuité des luttes pour l'émancipation sociale.

Effectivement, notamment à cause de l'endettement des États et du surendettement des ménages, la situation globale du capitalisme est devenue si grave que, comme le note encore François Chesnais, «le chômage, la dégradation du pouvoir d'achat et des systèmes de protection sociale et le maintien sinon l'aggravation des inégalités de rémunération et de patrimoine, créent les conditions pour consolider les bases de cet anticapitalisme qui sourd. Il s'agit de transformer ce sentiment en une volonté politique portée par des objectifs précis, simultanément économiques et écologiques. Le lien entre "l'irresponsabilité» des gouvernements et des groupes industriels face au changement climatique et les ressorts de la production capitaliste peut être démontré. Celui aussi entre les processus de dégradation accentuée des sols et des ressources et l'exploitation débridée du travail des femmes, des hommes et des enfant ${ }^{14}$. "

Il serait cependant naiff de penser que ces exigences sont faciles à réaliser, car elles touchent au nerf central du capitalisme. Leur application par un gouvernement de gauche présupposerait, entre autres, la socialisation des grands moyens de production, la nationalisation des banques et l'institution d'une véritable démocratie de base participative, garantissant au citoyen un droit de regard et de codécision réel dans les affaires politiques, précisément dans la perspective concrète d'une transition vers l'écosocialisme. Or, le Parti socialiste français n'est évidemment pas prêt à se rallier à ces objectifs. Il s'agit, pour les dirigeants de ce parti, essentiellement, de gouverner "mieux », en poursuivant plus ou moins la politique néo-libérale du gouvernement précédent, avec quelques petites réformes "cosmétiques", en ne faisant qu'un minimum absolu de concessions à leurs partenaires politiques d'Europe Écologie/Les Verts (EE/LV). Comme cela était déjà le cas à l'époque de Lionel Jospin, la « rupture avec le capitalisme » ne fait absolument pas partie du programme des socialistes.

Bien sûr, l'adversaire politique (l'oligarchie UMP), la haute finance et les élites technocratiques à son service ne tardent pas à qualifier ces propositions d'« utopiques " et d'«irréalistes", alors qu'elles ne sont que la réponse nécessaire aux problèmes écologiques, sociaux, politiques et moraux qui se posent avec urgence aujourd'hui.

Or la transformation progressive, intervenue dans les années 1970-1990, des partis socialistes et sociaux-démocrates en des formations politiques réformistes néo-capitalistes, le discrédit jeté en bloc sur le socialisme moderne par la déviation stalinienne et ultrabureaucratique du communisme autoritaire et totalitaire en URSS et dans ses pays satellites, sont bien les principales causes de la situation globalement difficile du socialisme en 
France et dans le monde, et des difficultés de ce courant de pensée politique pour se constituer de nouveau comme une grande force politique offensive crédible, capable d'assumer le changement, voire le rôle du principal acteur politique d'une transformation écologique et sociale radicale de nos sociétés libérales de consommation. C'est aussi la principale raison de la marginalisation du courant écosocialiste, qui a quand même pu se développer, au courant des années 1980 et 1990, principalement en réaction au courant de l'écologie profonde ("deep ecology») créé en 1973 par le philosophe norvégien Arne Naess, et qui a marqué pendant plusieurs décennies le mouvement écologiste en Europe Occidentale et en Europe Centrale.

Malgré ces difficultés, malgré cette marginalisation (par exemple la motion altermondialiste écologiste du courant «Utopia » n'avait obtenu au Congrès de Reims du Parti socialiste en 2008 que moins de $2 \%$ du vote des militants!), la tendance politique écosocialiste a pu, contre vents et marées, se développer et a même pu consolider ses positions, aux États-Unis, en Amérique du Sud et en Europe, durant les trois dernières décennies, grâce à l'élan de son internationalisme (cf. le Réseau Écosocialiste International) et à la productivité intellectuelle incontestable de ses principaux théoriciens (James O'Connor, Joël Kovel, René Dumont, André Gorz, Michael Löwy...). Globalement, ce courant écosocialiste s'est différencié, dans le courant des dernières décennies, en plusieurs tendances qui ont toutes en commun d'articuler le combat pour la préservation et la sauvegarde de la nature et de l'environnement, ainsi que le combat contre l'implantation des centrales nucléaires, avec les luttes sociales, à savoir les diverses formes de lutte pour l'émancipation des exploités et des aliénés, contre la discrimination des minorités ethniques, des femmes, etc. Mais des nuances importantes distinguent ces tendances entre elles. Elles ont pourtant comme dénominateur commun de s'opposer à d'autres courants de l'écologie (politique) et notamment au courant de l'écologie profonde, aux partisans d'une écologie réformiste et à ceux d'une économie écologique capitaliste.

$[\ldots]$

\section{La contribution d'André Gorz à la critique de l'écologie politique (pp. 94-101)}

Tandis que, sur le front politique, après l'échec de René Dumont aux élections présidentielles, le mouvement écologiste français s'était restructuré, entre 1974 et 1980, sous l'égide des «Amis de la Terre » (Antoine Waechter, Brice Lalonde...) et d'un grand nombre d'associations environnementalistes, la publication, en 1975, du livre Écologie et Politique par André Gorz ${ }^{15}$ changea la donne, par sa réactualisation originale des principales revendications écologistes, et en donnant en même temps à ce mouvement un nouvel élan théorique. Pour André Gorz lui-même, qui s'était fait connaître, en France, pour la première fois, en 1958, avec son roman très «sartrien" Le Traitre ${ }^{16}$, ce livre marquait aussi le tournant d'une pensée existentialiste - fortement influencée par Sartre et, ultérieurement, aussi par les idées du socialisme autogestionnaire (PSU/Il Manifesto) -, vers une nouvelle pensée écologique influencée de manière déterminante par Ivan Illich. Partageant avec René Dumont la critique du nucléaire (qu'il appelait le «fascisme électrique »), du productivisme et du consumérisme, ainsi que l'idée que nous nous trouvions déjà au seuil de la naissance d'une nouvelle civilisation, Gorz articule sa propre critique du capitalisme avancé avec les critiques radicales faites par Illich, au système de santé, etc. 
Dans ses premiers livres, Stratégie ouvrière et néo-capitalisme (1964), Le socialisme difficile (1967) et Réforme et Révolution (1969), Gorz se positionnait encore - notamment avec sa théorie du "réformisme-révolutionnaire »- comme un théoricien et un intellectuel marxiste de la gauche de la gauche, ayant été très influencé, comme avant lui René Dumont, par la théorie de l'autogestion du PSU (Il Manifesto). Après son tournant vers l'écologie, ce premier engagement théorique ne sera pas complètement abandonné ; il se manifestera encore notamment sous la forme d'un anticapitalisme et de l'assignation au capitalisme de la responsabilité de la crise écologique. En 1980, il se permet cependant, avec la publication de son livre Adieux au prolétariat? Au-delà du socialisme, quelques critiques de l'orthodoxie marxiste, en reformulant la critique du stalinisme et du bureaucratisme étatique de l'URSS et des pays du « socialisme réellement existant ", tout en rejetant le réformisme de la social-democratie, et en réaffirmant les valeurs d'un socialisme authentiquement démocratique.

Dans le cadre de cette critique, il va jusqu'à mettre en cause certains dogmes sacro-saints du néo-marxisme contemporain (dans ses diverses composantes), en particulier à propos de la théorie du prolétariat comme principal sujet des transformations économicosociales. Le prolétariat ne peut plus être considéré, selon Gorz, comme le seul et unique sujet de la révolution sociale à venir, telle que Marx l'avait enseigné, parce que dans les conditions de l'automatisation et de la robotisation (progressive) de la production industrielle du capitalisme avancé, ce qui était autrefois la "classe des prolétaires » ne possède plus selon lui la conscience révolutionnaire qu'avaient les travailleurs industriels des entreprises à l'époque de l'accumulation forcée du Capital. Par conséquent, cette classe qui, selon Marx et les "marxistes" du $\mathrm{xx}^{\mathrm{e}}$ siècle, devait toujours se trouver à l'avant-garde du combat pour l'émancipation des travailleurs de l'aliénation et de l'exploitation, ne peut plus jouer ce rôle. Elle a été progressivement remplacée, en raison de toute une série de transformations technologiques et sociales intervenues dans la seconde moitié du $\mathrm{xx}^{\mathrm{e}}$ siècle, par une sorte de "non-classe des néo-prolétaires ${ }^{17}$ " constituée par des travailleurs occasionnels, des chômeurs, des précaires, des marginalisés, etc. André Gorz se démarque ainsi de Marx et des marxistes en prophétisant l'avènement inéluctable d'une société sans travail ${ }^{18}$, précédée sans nul doute d'une période transitoire marquée par une très forte réduction du travail hebdomadaire jusqu'à 20 heures (!), et en revendiquant l'institution d'un revenu d'existence. Car, sans nul doute mieux que tout autre, Gorz avait annoncé la « révolution du temps libre » résultant du nombre toujours croissant d'hommes et de femmes vivant en dehors du processus de production, des usines et des bureaux, suscitant, entre autres, le problème de la gestion des loisirs. Faisant preuve d'une force visionnaire tout à fait exceptionnelle, André Gorz était aussi l'un des tout premiers théoriciens critiques à remarquer la transformation du capitalisme avancé en un capitalisme financier de la spéculation bancaire (opérant de plus en plus avec du capital fictif) et en un capitalisme cognitif fondé sur une économie de l'immatériel. Cela lui a également permis d'anticiper la crise des «subprimes » d'octobre 2008 aux États-Unis et ses conséquences néfastes pour le système bancaire mondial...

En redéfinissant l'écologie comme une "éthique de la libération ${ }^{19}$ ", Gorz était parvenu, dans ses tout derniers écrits, à la conviction que la sortie du capitalisme avait en réalité déjà commencé : "par son développement même le capitalisme a atteint une limite tant interne qu'externe qu'il est incapable de dépasser et qui en fait un système qui survit par des subterfuges à la crise de ses catégories fondamentales: le travail, la valeur, le capital ${ }^{20}$. " Avec le recyclage bancaire de plus-values fictives, l'économie réelle devient, affirme-t-il en 2007, «un 
appendice des bulles spéculatives entretenues par l'industrie financière. Jusqu'au moment inévitable, où les bulles éclatent, entraînant les banques dans des faillites en chaîne, menaçant le système mondial de crédit d'effondrement, et l'économie réelle d'une dépression sévère et prolongée (la dépression japonaise dure déjà depuis bientôt quinze ans) ${ }^{21}$. »Croyant comme Illich dans le potentiel inépuisable des ressources humaines, de la coopération et de la créativité humaine, Gorz semble aussi être persuadé que cet "excédent des ressources» humaines pourrait devenir productif dans une économie autre « où la création de richesses n'est pas soumise aux critères de rentabilité. " Autrement dit, dans une économie écologique, sociale et solidaire, où tout serait produit dans des ateliers coopératifs ou communaux, et « où les activités de production pourront être combinées avec l'apprentissage et l'enseignement, avec l'expérimentation et la recherche, avec la création de nouveaux goûts, parfums et matériaux, avec l'invention de nouvelles formes de technique d'agriculture, de construction, de médecine, etc. Les ateliers communaux d'autoproduction seront interconnectés, à l'échelle du globe, et pourront échanger ou mettre en commun leurs expériences, inventions, idées, découvertes. Le travail sera producteur de culture, l'autoproduction d'un mode d'épanouissement ${ }^{22}$.» Ce ne sont pas là les rêveries utopiques abstraites d'un "visionnaire", mais des propositions concrètes qui sont partiellement mises en œuvre, notamment avec la création des «Fablabs", aux États-Unis. Comme le souligne Jérôme Gleize, "ces ateliers composés de machines-outils pilotées par ordinateur, pouvant fabriquer à la demande des biens de nature variée et singulière, sont une alternative à l'usine, productrice de biens uniformes de masse ${ }^{23}$. » Leur généralisation pourrait donc bien inaugurer une révolution du mode de production capitaliste «traditionnel» et son remplacement par un mode de production autonome, autogestionnaire, décentralisé et démocratique.

\section{NOTES}

1. D. Tanuro, L'Impossible capitalisme vert, préface de M. Husson, Paris, La Découverte, 2010. Daniel Tanuro est le fondateur belge de l'ONG « Climat et justice sociale ».

2. Ibid., p. 212.

3. M. Horkheimer / T. Adorno, Dialectique de la Raison. Fragments philosophiques, trad. de l'allemand par Eliane Kaufholz, Paris, Gallimard, 1974 ; cf. M. Horkheimer, L'Eclipse de la raison, 1951.

4. D. Tanuro, op. cit.

5. D. Tanuro, op. cit., p. 10.

6. F. Chesnais, «Écologie, luttes sociales et projet révolutionnaire pour le XxI ${ }^{\mathrm{e}}$ siècle ", in V. Gay (coord), Pistes pour un anticapitalisme vert, Paris, Syllepse, 2010, p. 19.

7. Groupe d'experts intergouvernemental sur l'évolution du climat.

8. Ibid., p. 8.

9. D. Tanuro, op. cit., p. 244.

10. Comme le souligne à juste titre à ce propos Robert Kurz, cette situation a soudainement remis à l'honneur "des éléments centraux du keynésianisme (étatisation ou nationalisation des banques et, éventuellement d'industries clé, régulation des marchés financiers", mais, "contrairement à la fin de l'époque fordiste dans les années 1970, nous n'avons plus affaire au keynésianisme de l'État-providence, mais à celui de l'État d'urgence capitaliste financier qui accompagne l'aggravation de la gestion antisociale 
par l'État du travail et des individus. C'est la prolongation paradoxale du néolibéralisme avec des moyens quasi keynésiens... » (R. Kurz, Vies et mort du capitalisme. Chroniques de la crise, Nouvelles Éditions Lignes, Fécamp, 2012, p. 153.)

11. F. Chesnais, op. cit., p. 21.

12. Cf. H. Jonas, Le Principe Responsabilité (Une éthique pour la civilisation technologique), trad. J.Greisch, Le Cerf, Paris, 1990; cf. aussi: A. Münster, Principe Responsabilité ou Principe Espérance? (H. Jonas, G. Anders, E. Bloch), Le Bord de l'eau, Lormont, 2011.

13. F. Chesnais, op. cit., p. 23.

14. F. Chesnais, op. cit., p. 24.

15. En ce qui concerne l'œuvre théorique d'André Gorz, on peut se référer aux textes suivants : A. Münster, André Gorz ou le socialisme difficile, Paris, Nouvelles Éditions Lignes, 2008 ; C. Fourel (dir.), André Gorz: un penseur pour le XXI siècle, Paris, La Découverte, 2009 ; «Repenser le travail avec André Gorz », Ecorev n 28, novembre 2007 ; «Penser l'après-capitalisme avec André Gorz », Ecorev n 33 novembre 2009 ; Arno Münster, «André Gorz, "dérangeur” ou constructeur d'une nouvelle utopie écosocialiste » ?, in Ecorev n 33, Paris, novembre 2009, p. 82-87.

16. Cf. A. Gorz, Le Traître, Avant-Propos de J-P. Sartre, Paris, Le Seuil, 1958, rééd. Gallimard, FolioEssais, 2005.

17. A. Gorz, Adieu au prolétariat ? Au-delà du socialisme, Paris, Galilée, 1980.

18. Cf. A. Gorz, Métamorphoses du travail. Quête du sens. Critique de la raison économique, Paris, Galilée, 1988, rééd. Gallimard « Folio-essais », 2004. Cf. aussi A. Gorz, «Penser l'exode de la société de travail et de la marchandise », « Mouvements » $n^{\circ} 50$, juin 2007.

19. Cf. l'entretien de Marc Robert avec André Gorz in Ecorev n ${ }^{\circ} 28$, novembre 2007, p. 83-87.

20. A. Gorz, "Le travail dans la sortie du capitalisme " alias "La sortie du capitalisme a déjà commencé ", Ecorev n ${ }^{\circ} 28$ (novembre 2007).

21. A. Gorz, «Le travail dans la sortie du capitalisme », in Ecorev n ${ }^{\circ} 28$, novembre 2007, p. 10.

22. Ibid., p. 14.

23. J. Gleize, «Le potentiel subversif des FabLabs comme mode de production », in Ecorev n ${ }^{\circ}$ 37, Paris, août 2011, p. 78-81.

\section{INDEX}

Mots-clés : critique du travail, correspondance, artistes, socialisme vert, travailleurs culturels, Wertkritik, Gorz André, dialogue, Vincent Jean-Marie, écologie, modèles alternatifs, Marx Karl, penser le Capital, ethos du travail, Eglise Orthodoxe, salariat, domination

\section{AUTEUR}

\section{ARNO MÜNSTER}

Historien de la philosophie moderne et contemporaine, maître de conférences de philosophie à l'Université de Picardie Jules Verne d'Amiens. 